\title{
Verticilliose de la tomate: expressions de la résistance générale chez deux lots d'origine
} sauvage

\author{
Issa BÉYE \& Jean-François LAFAY \\ Laboratoire de Cryptogamie associé au C.N.R.S. (UA.086), Université Paris-Sud, Bâtiment 400, F91405 Orsay \\ Cedex
}

\begin{abstract}
Différentes expressions de la résistance générale ont été étudiées chez un représentant de chacune des 2 espèces sauvages de tomate: L. pimpinellifolium et L. esculentum var. cerasiforme. Aucun des 2 lots n'a manifesté un comportement optimum vis-à-vis de chacune des expressions de la résistance générale. L. pimpinellifolium a présenté des caractéristiques intéressantes pour la durée de la période d'incubation, l'intensité des altérations foliaires et leur extension sur l'axe aérien, mais le rythme de l'émission des feuilles est fortement dérégulé L. esculentum var. cerasiforme ne présente pas non plus de désordres foliaires graves mais ces derniers se manifestent après une période d'incubation nettement plus courte et progressent en direction de l'apex sans être freinés ; en revanche, l'émission des feuilles n'est pas perturbée par la maladie.

Aucun des 2 lots d'origine sauvage ne s'est montré protégé vis-à-vis des perturbations de la croissance de l'axe aérien et de l'émission des fleurs.

Cette étude, bien que limitée, laisse supposer que l'amélioration de l'ensemble des expressions de la résistance générale de la tomate exigerait de cumuler des éléments avantageux dispersés à la fois dans les espèces sauvages et dans les multiples variétés de tomate cultivées.
\end{abstract}

Mots clés additionnels : Symptômes, Verticillium dahliae, Lycopersicon pimpinellifolium (Jusl.) Mill., Lycopersicon esculentum var. cerasiforme (Dun.) Mill.

Various expressions of general resistance were studied in representatives of 2 wild-type tomato species: L. pimpinellifolium and L. esculentum var. cerasiforme. Neither group exhibited optimal behaviour towards all aspects of the disease. L. pimpinellifolium showed interesting characteristics with respect to incubation period and amount and spread of leaf damage, but leaf formation was greatly deregulated. Leaf damage inflicted to L. esculentum var. cerasiforme was not serious either, but occurred after a much shorter incubation period and moved unhampered towards the apex. The disease did not, however, disturb leaf formation significantly. Neither group showed immunity towards disturbances of stem growth and flower formation. Although the limited sample involved in this study does not reflect the great diversity of wild-type species, the results indicate that the improvement of general resistance would require the accumulation of favourable elements presently scattered throughout wild-type species as well as the numerous varieties of cultivated tomatoes.

Additional key words : Symptoms, Verticillium dahliae, Lycopersicon pimpinellifolium (Jusl.) Mill., Lycopersicon esculentum var. cerasiforme (Dun.) Mill.

\section{INTRODUCTION}

La domestication de la tomate, en orientant la sélection vers un petit nombre d'objectifs étroitement définis, peut n'avoir pas pris en compte toutes les potentialités des espèces sauvages. Ceci expliquerait que les divers symptômes de la verticilliose induits par un même clone de Verticillium dahliae Kleb. revêtent des formes et des intensités différentes selon les variétés cultivées contaminées (BEYE \& LAFAY, 1985). En effet, ces variétés cultivées peuvent ne pas avoir reçu de leurs ancêtres l'ensemble des éléments de leur patrimoine génétique 
qui les protègent contre la verticilliose et les éléments transmis peuvent différer d'une variété à l'autre. La vérification de cette hypothèse pourrait conduire à une réorientation des programmes de sélection pour la résistance à la verticilliose. C'est pourquoi, nous avons étudié le comportement de 2 représentants de formes ancestrales de la tomate cultivée.

\section{MATÉRIEL ET MÉTHODES}

L'expérimentation a porté sur des plantes issues de semences de Lycopersicon esculentum var. cerasiforme répertorié «WVa 106 » et de L. pimpinellifolium répertorié «WVa 700 ». Il s'agit de populations recueillies dans la nature, mais dont la polymorphie a peut-être été réduite par la multiplication en station. Vis-à-vis de la verticilliose, l'espèce $L$. pimpinellifolium est celle qui a fourni le gène $V e$ de résistance à la race commune de $V$. dahliae. Ainsi que nous le discuterons plus loin, nous pensons que le gène $V e$ est présent chez «WV 700 » et absent chez «WVa 106 ». Les conditions de culture, le mode d'inoculation et la notation des effets de la verticilliose sont ceux décrits par BEYE \& LAFAY (1985). Les expériences ont été conduites sur 88 plantes issues de chacun des 2 lots de semences. La moitié des 88 plantes a été contaminée, l'autre moitié servant de témoin. Le clone de $V$. dahliae $C 19$ obtenu par SeBTi (1982) est utilisé. Les expressions de la maladie retenues sont : la durée de la période d'incubation, la progression cles symptômes foliaires sur la tige en direction de l'apex, l'intensité des altérations foliaires, les perturbations de la croissance de l'axe aérien, de l'émission des feuilles et des fleurs. Ces différents paramètres ont été définis par BEYE \& LAFAY (1985).

Pour celles de ces expressions dont l'intensité varie au cours du temps, des observations ont été effectuées chaque semaine pendant 6 semaines à partir de la fin de la période d'incubation.

Le logiciel statistique SPSS (Statistical Package for the Social Sciences) a été utilisé pour l'analyse des résultats (Univac 1110 , Centre Paris-Sud Informatique).

\section{RÉSULTATS}

\section{A. Durée de la période d'incubation}

Les premiers signes foliaires de la maladie (épinastie, flétrissement) apparaissent chez «WVa 700 " après un temps d'incubation $(15,5 \pm 0,6$ jours $)$ nettement plus long que chez «WVa $106 »(10,1 \pm 0,2$ jours $)$.

\section{B. Intensité et extension des symptômes foliaires (fig. 1)}

Chez «WVa 700 », les symptômes foliaires consistent principalement en de légers brunissements. Ces altérations progressent en direction de l'apex à une vitesse inférieure à celle de l'émission des feuilles. Aussi l'indice d'altération foliaire marque-t-il une nette décroissance à

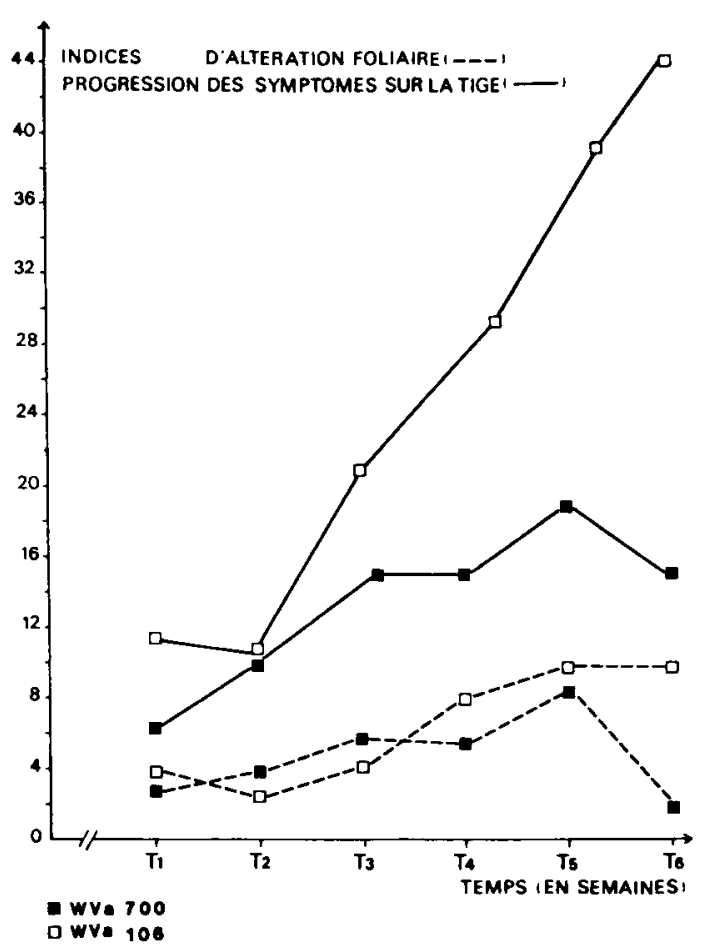

Figure 1

Evolution de l'indice d'altération foliaire (en p. 100 de l'altération maximum possible), et progression des symptômes foliaires (hauteur atteinte, en p. 100 de la hauteur totale de la plante).

TI à T6: nombre de semaines après la fin de la période d'incubation.

Index of leaf symptoms (\% of maximum possible damage), and spread of leaf symptoms (height reached, as \% of total plant height).

TI to T6: number of weeks after the end of the incubation period.

la fin de la période d'observation. En outre, les dommages ne sont jamais étendus au-delà du cinquième de la hauteur des tiges.

Chez «WVa 106 », les brunissements évoluent en nécroses; ces altérations foliaires demeurent de peu d'importance mais progressent sans interruption vers le haut et atteignent en fin d'expérience la moitié de la plante.

\section{Croissance de l'axe aérien (fig. 2)}

Les perturbations de la croissance provoquées par l'inoculation du clone $C 19$ de $V$.dahliae sont très profondes chez «WVa $700 »$. Un rabougrissement important est observé dès les premières manifestations foliaires de la maladie. Cette réduction de la taille persiste jusqu'à la quatrième semaine après la fin de la période d'incubation. A ce moment, la croissance de la tige s'accélère au point de compenser le retard et de surpasser les témoins en l'espace d'une semaine.

Chez «WVa 106 » une dépression initiale de la croissance de l'axe aérien est également notée. Le rabougrissement, peu marqué au début, s'accentue progressivement jusqu'à un déficit en hauteur de 22 p. 100 par rapport aux témoins, 4 semaines après la fin de la période d'incubation. L'élongation de l'axe aérien des plantes contaminées s'accélère ensuite mais sans compenser totalement, du moins jusqu'à la fin de nos observations, le retard accumulé. 


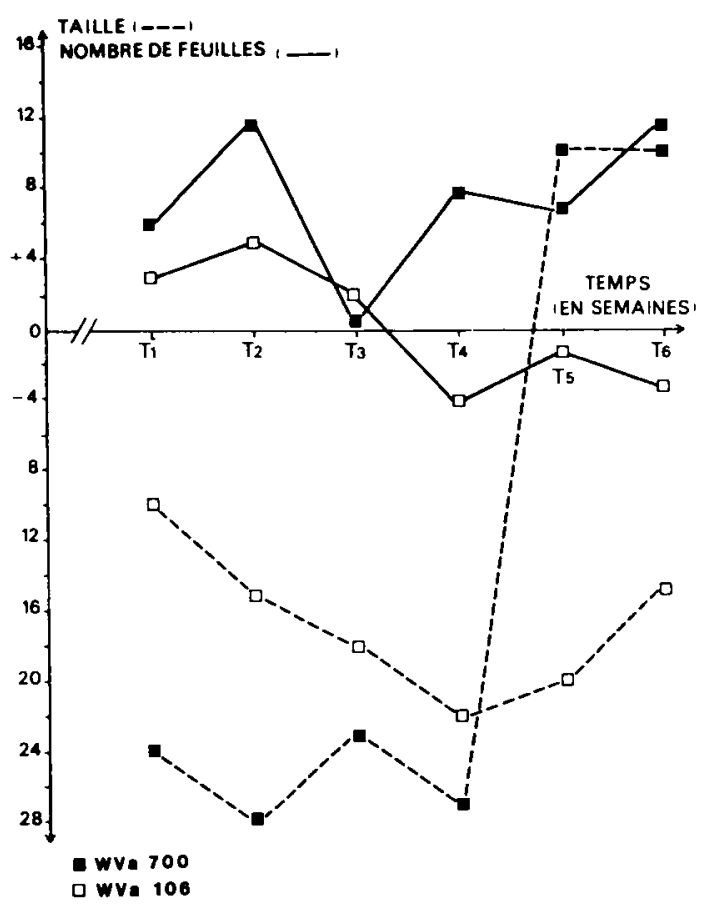

Figure 2

Evolution de la taille moyenne des plantes et du nombre moyen de feuilles émises, en p. 100 du témoin.

TI à T6: nombre de semaines après la fin de la période d'incubation.

Average plant height and leaf number, as \% of control.

TI to T6: number of weeks after the end of the incubation period.

\section{Emission des feuilles (fig. 2)}

La contamination de "WVa 700 » entraîne un accroissement du nombre des feuilles émises presque sans interruption pendant toute la durée de l'expérience. En revanche, cette activité n'est pas perturbée chez «WVa 106 »: les écarts par rapport aux témoins ne sont pas statistiquement significatifs.

\section{E. Emission des fleurs}

Le nombre de fleurs constituant les 3 premières inflorescences est, chez les 2 espèces, diminué de manière analogue par l'inoculation de $V$.dahliae. Chez «WVa 700 » ce nombre passe de 11,3 $\pm 0,8$ fleurs chez les plantes témoins à $9,4 \pm 0,9$ fleurs chez les plantes inoculées; cette différence est significative au seuil de 5 p. 100 . La différence entre témoins et inoculées est également significative chez "WVa 106 "; les nombres sont respectivement $11,6 \pm 0,9$ et $8,7 \pm 0,9$ fleurs.

\section{DISCUSSION ET CONCLUSION}

L'échantillon "WVa 700 " exprime sous diverses formes un bon niveau de résistance générale: une période d'incubation longue, un indice d'altération foliaire particulièrement bas, une progression très lente des symptômes foliaires vers le sommet de la plante. Néanmoins, l'accélération de l'émission des feuilles est notable. L'échantillon «WVa 106 »'est comporté différemment de "WVa 700 » pour une partie des éléments de résistance générale analysés : les premiers symptômes apparaissent plus rapidement, les altérations foliaires évoluent en nécroses et, tout en demeurant légères, progressent de manière continue vers l'apex. L'émission des feuilles n'est pas altérée par la maladie.

Les 2 lots étudiés se sont comportés de façon analogue quant à leur sensibilité vis-à-vis des perturbations de l'élongation de l'axe aérien et de l'émission de fleurs. Chez "WVa 700 », la dérégulation de la croissance de l'axe aérien est marquée; le rabougrissement des tiges de "WVa 106 » est net et durable. La formation des fleurs est sensiblement altérée dans les 2 populations.

La sensibilité des espèces sauvages à la composante du pouvoir pathogène de Verticillium dahliae qui perturbe la croissance de l'axe aérien n'est pas une observation nouvelle. SCHAIBLE et al. (1951), en étudiant l'utilisation d'une espèce sauvage en provenance du Pérou comme géniteur du gène $V e$ de résistance à la race 1 de $V$. dahliae ont déjà signalé un rabougrissement de certaines plantes inoculées, mais comme ils ont observé le même phénomène chez certains témoins, ils ne l'ont pas considéré comme un effet de la maladie. Parmi les variétés cultivées dont les réponses à l'inoculation du clone $C 19$ de $V$. dahliae ont été précédemment étudiées (BEYE \& LAFAY, 1985), la variété " Roma VF » (qui porte le gène $V e$ ) rappelle par plusieurs traits les comportements de «WVa 700 » : la présence du gène $V e$ ne la protège pas totalement mais les désordres engendrés par la maladie sont, pour la plupart, peu accusés. Ce rapprochement avec "Roma VF", ainsi que le fait que l'espèce L.pimpinellifolium, dont est issu «WVa 700 », soit à l'origine du gène $V e$ en sélection, nous font penser que "WVa 700 » possède probablement ce gène $V e$, lequel, de toute façon, ne protège pas complètement vis-à-vis de la verticilliose induite par le clone C19. Par contre, compte tenu des différences de comportement évoquées plus haut, nous estimons que "WVa 106 " ne possède pas $V e$.

D'autres variétés cultivées notamment "Raf» et "Supermarmande" plus sensibles à la verticilliose se comportent aussi bien que "WVa 700 " et nettement mieux que "WVa 106 » à en juger par certaines des expressions de la résistance générale telles que la longueur de la période d'incubation et la sensibilité aux perturbations de l'axe aérien. Pour ces caractéristiques, il n'y a pas d'amélioration à attendre d'un retour aux 2 populations sauvages que nous avons utilisées. En revanche, les 2 lots sauvages se sont montrés mieux protégés que les variétés cultivées "Raf", "Supermarmande " et "Red Jacket " vis-à-vis des effets du parasite sur le fonctionnement des feuilles au niveau des altérations foliaires qui sont réduites. Pour cette dernière caractéristique au moins, un retour aux sources de résistance que recèlent les espèces sauvages pourrait entraîner une amélioration appréciable.

Ainsi, cette étude, bien que très ponctuelle parce que menée sur un échantillonnage restreint de 2 populations, conduit à penser que l'obtention d'une bonne résistance générale exigerait de cumuler des éléments 
avantageux dispersés à la fois dans les espèces sauvages et dans les multiples variétés de tomate cultivées.

Reç le 21 juin 1987. Accepté le 3 mars 1988.

\section{REMERCIEMENTS}

Les auteurs remercient Monsieur le Professeur Chevaugeon pour ses nombreux avis et conseils dans la préparation et la réalisation du texte. Ils remercient également Monsieur TAILlER pour son aide technique ainsi que Monsieur LATERROT pour la fourniture des semences.

\section{RÉFÉRENCES BIBLIOGRAPHIQUES}

Beye I., Lafay J. F., 1985. Etude de critères de sélection pour une résistance générale à la verticilliose chez la tomate. Agronomie, 5, 305-311.

Schaible L., Cannon O. S., Waddoups V., 1951. Inheritance of resistance to verticillium wilt in a tomato cross. Phytopathology, 41, 986-990

Sebti S., 1982. Essai d'analyse des composantes du pouvoir pathogène de Verticillium albo-atrum Reinke et Berthold. Thèse Doct. $3^{\mathrm{c}}$ Cycle, Univ. Paris-Sud, Orsay. 93 p. 